\title{
Chondromesenchymal Hamartoma in Ectopic Thyroid Tissue in a Neonate
}

\author{
Agnes Szepesi ${ }^{1}$ Zsolt Juhasz ${ }^{2}$ Anna Kover ${ }^{2}$ Bela Kajtar $^{3}$ Noemi Benedek ${ }^{2}$ Endre Kalman ${ }^{3}$ \\ Tamas Kovesi $^{4}$ Marianna Imre ${ }^{5}$ Peter Vajda ${ }^{2}$
}

${ }^{1}$ Department of Pediatric Surgery, Petz Aladar County Teaching Hospital, Gyor, Hungary

2 Department of Pediatrics, University of Pecs, Medical Center, Pecs, Hungary

${ }^{3}$ Department of Pathology, University of Pecs, Medical Center, Pecs, Hungary

${ }^{4}$ Division of Pediatric Anesthesiology, Department of Anesthesiology, University of Pecs, Medical Center, Pecs, Hungary

${ }^{5}$ Diagnostic Center of Pecs, Hungary

Eur J Pediatr Surg Rep 2019;7:e39-e42.

\begin{abstract}
Address for correspondence Agnes Szepesi, MD, Department of Pediatric Surgery, Petz Aladar County Teaching Hospital, 2-4 Vasvary Pal Street, Gyor 9023, Hungary (e-mail: md.szepesiagi@gmail.com).
\end{abstract}
Abstract
Keywords
- chondrome- senchymal
- hamartoma
- thyroid
- ectopic

A full-term male neonate presented with a left sided cervical lump at the level of the thyroid gland. Magnetic resonance imaging (MRI) showed a benign heterogeneous solid mass with lobulated margins. The tumor underwent complete excision. Histology revealed the diagnosis of chondromesenchymal hamartoma in ectopic thyroid tissue.

\section{New Insights and the Importance for the Pediatric Surgeon}

The authors report the first case of chondromesenchymal hamartoma arising in ectopic thyroid tissue in neonate.

\section{Introduction}

Head and neck tumors are rare entities in neonates. They mainly consist of vascular malformations, other developmental lesions, and rarely other benign or malignant soft tissue tumors. ${ }^{1}$

Cystic hygromas on the neck are the most common vascular malformations. They are classically found in the left posterior triangle of the neck. Large cystic hygromas can cause fetal hydrops and neonatal respiratory difficulties. ${ }^{2} \mathrm{Ex}$ utero intrapartum treatment (EXIT) procedure can be performed for urgent management of severe airway obstructions caused by the detected giant neck mass. ${ }^{3}$

We report on a neonate with cervical mass which turned out to be a hamartoma arising from ectopic thyroid tissue.

\section{Case Report}

A full-term 1-day-old male neonate with a birth weight of 3,420 g was admitted to our department with a left sided cervical mass. The patient had a history of fetal tachycardia and maternal fever during the pregnancy. However, the cervical lesion was not detected on antenatal ultrasounds.

On physical examination, the patient had a painless mobile mass on the left side of the neck at the level of the thyroid gland, 3 to $4 \mathrm{~cm}$ in diameter ( - Fig. 1).

Otherwise no abnormality was detected.

On magnetic resonance imaging (MRI) a large mass was seen in the soft tissue of the neck extending from the left side to the midline. The heterogeneous enhancing lobulated tumor received

September 7, 2018

accepted after revision

March 31, 2019

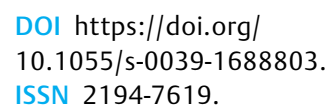

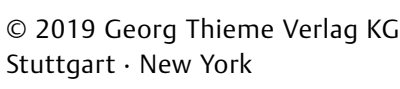

License terms

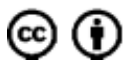




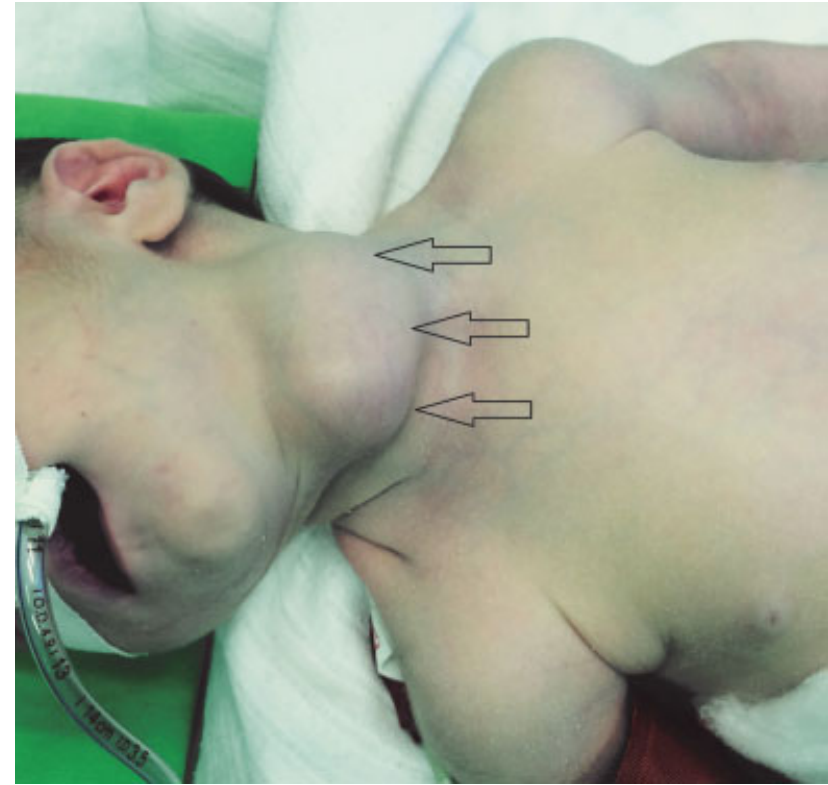

Fig. 1 Solid mass on the neck extending from the left side to the midline (arrows).

slightly dislocated the hypopharynx, larynx, and trachea, causing mild tracheal dislocation and compression. The major vessels of the neck were also mildly dislocated. The morphology of the mass on MRI was not specific for any type of tumor. The thyroid gland was of normal size, shape, and structure. No pathologic lymph nodes or signs of invasion of surrounding tissues were detected ( $\sim$ Fig. $2 \mathrm{~A}-\mathrm{C}$ ).

On the 10th day of life, the tumor underwent complete removal via Kocher's incision. The excision of the tumor was straightforward; the tumor was excised within its capsule without any injuries of the cervical structures ( - Fig. 3).

The perioperative period was uneventful. No recurrence was detected during the 6-month follow-up.

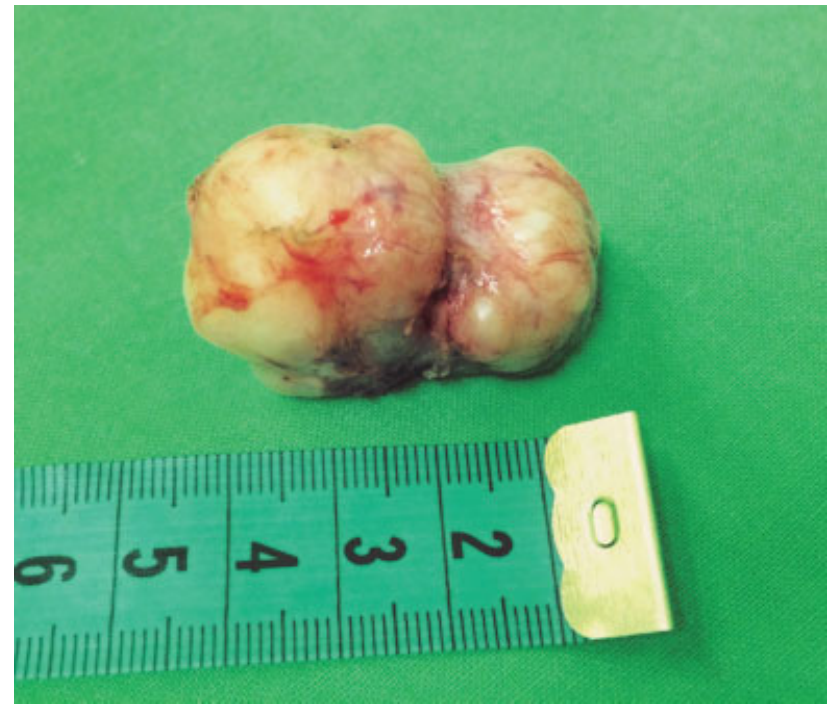

Fig. 3 The completely removed tumor.

\section{Pathological Finding}

On gross examination, the $1 \mathrm{~cm}$ mass was lobulated, firm in consistency, gray-white in color, and well demarcated.

Microscopic examination revealed a cell-rich, immature mesenchymal tissue with spindle cells, and nodules of hypercellular hyaline cartilage without evidence of malignancy. Scarce mitotic activity (1/10 high power fields) was noted in the spindle cell component which demonstrated collagen fibers and myxoid areas. Focally thyroid follicles were noted within the mass. The spindle cells were S100, smooth muscle actin (SMA), neurofilament (NF), and paired box gene 8 (PAX8) all negative, but showed vimentin and weak thyroid transcription factor 1 (TTF1) staining. The thyroid follicles showed cytokeratin (CK), PAX8, and TTF1 expression ( - Fig. 4A-D).
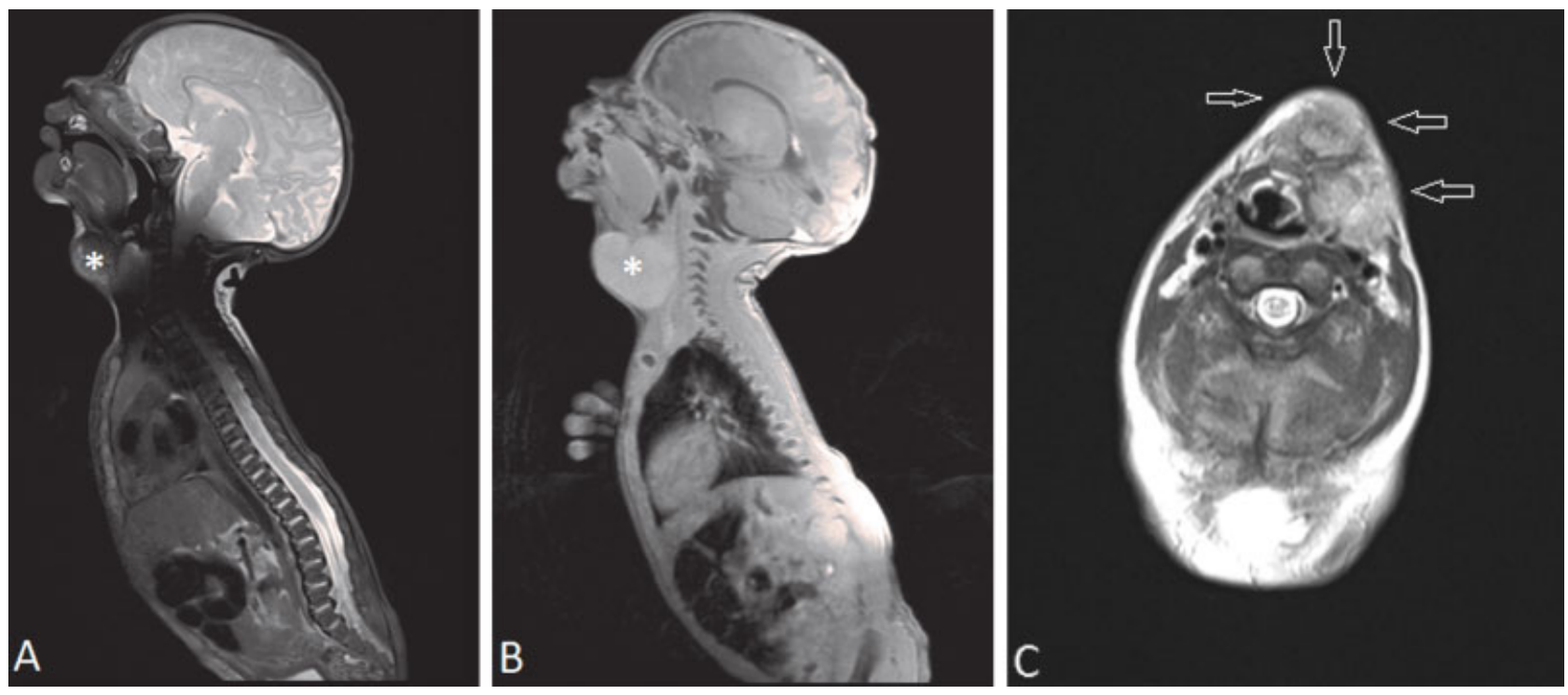

Fig. 2 (A, B) Sagittal T2 (A) and T1-weighted (B), MRI images, showing a lobulated tumor (asterisks) at the level of the thyroid gland, extending from the left side to the midline. (C) Axial, T2-weighted MRI demonstrates a tumor (arrows) on the left side of the neck at the level of the thyroid gland, dislocating the hypopharynx, larynx, trachea, and major vessels. 


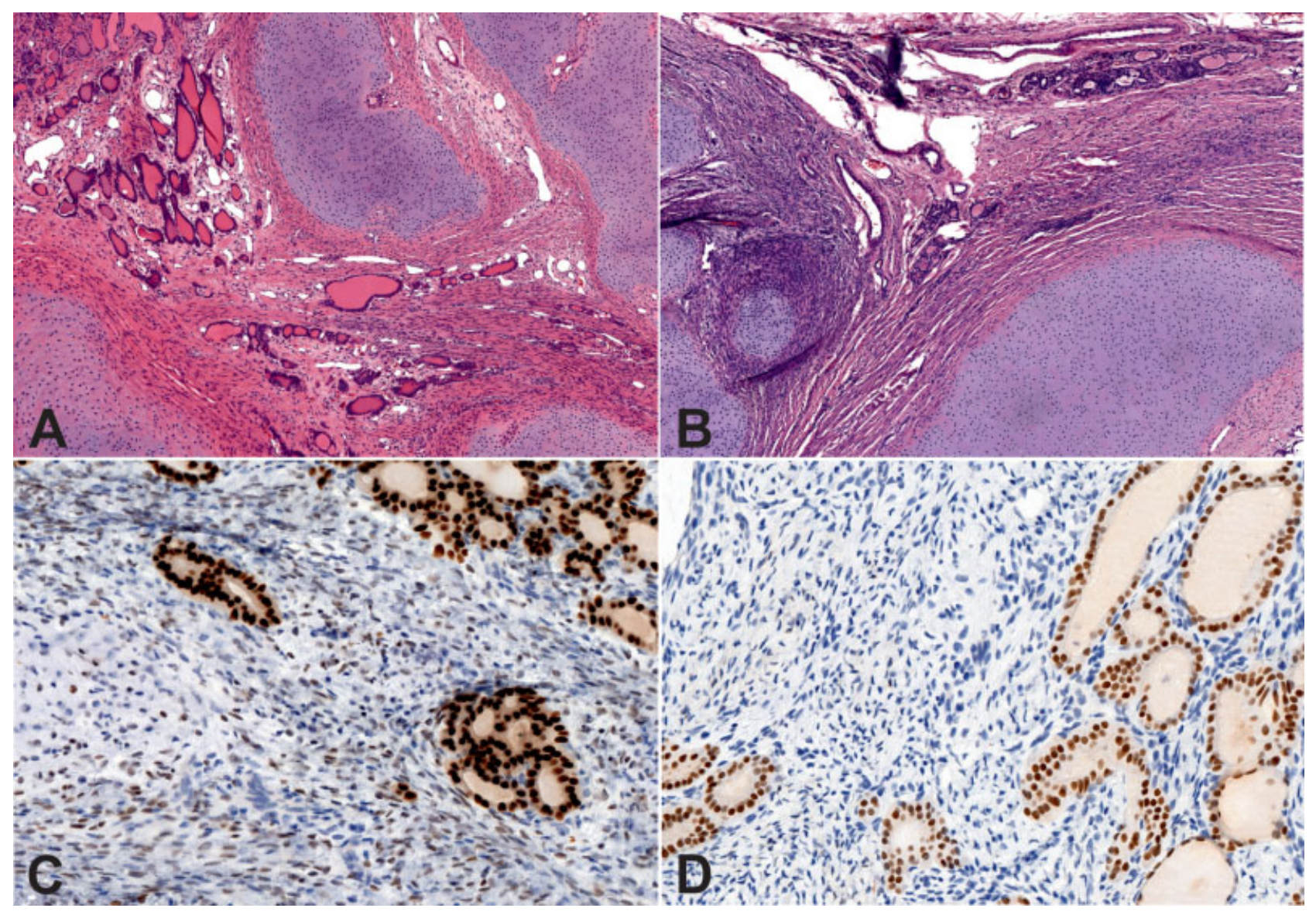

Fig. 4 Histology of the specimen. (A, B) Large lobules of hyaline cartilage are visible separated by spindle cells and scattered thyroid follicles $(\times 5$ magnification, hematoxylin-eosin staining). (C) Thyroid follicles and spindle cells show strong and weak TTF1 staining, respectively $(\times 20$ magnification). (D) Only thyroid follicles show PAX8 staining ( $\times 20$ magnification).

The findings were consistent with a chondromesenchymal hamartoma arising in ectopic thyroid tissue.

\section{Discussion}

The differential diagnosis of neck masses in children is based on clinical, pathological, and imaging characteristics. In childhood, most lesions are either congenital or inflammatory in origin and only a few neoplasms occur in the neck. The most common congenital developmental masses in the neck include thyroglossal duct cysts, branchial cysts, dermoid cysts, and vascular malformations. Inflammatory lesions can be a result of infectious lymphadenitis, reactive lymphadenopathy, or Kawasaki's disease. The differential diagnosis for neoplastic neck masses include benign tumors, such as haemangiomas, teratomas, plexiform neurofibromas, juvenile nasopharyngeal angiofibromas (JNA), and Langerhans cell histiocytosis (LCH), as well as malignant tumors, such as Hodgkin's lymphoma (HL) and nonHodgkin's lymphomas (NHL), rhabdomyosarcoma (RMS), thyroid malignances, nasopharyngeal carcinomas (NPC), salivary gland tumors, neuroblastoma, and metastasis. ${ }^{4-6}$

Teratoma is a subtype of germ cell tumors (GCT) derived from more than one of the three germinal layers. They are more common in the testes and ovaries, but can present in many different regions in the midline, including the sacral region, retroperitoneum, mediastinum, and brain. Mature teratomas are generally bening, immature teratomas in young children also tend to behave as benign tumors. In patients, older than 15 years, immature teratomas can manifest as highly devastating malignancies. ${ }^{7}$

Hamartomas are benign congenital nonneoplastic abnormalities, but can be locally aggressive leading to complications. Ectopic thyroid tissue is another congenital developmental defect that may present as a tumor. The prevalence is 1 in 100,000 to 300,$000 ; 90 \%$ of cases are localized to the base of the tongue. However, rare cases may appear in the cervical region resulting from abnormal migration and fusion of the lateral thyroid anlage. ${ }^{8}$

Chondromesenchymal hamartomas are rare lesions, most occur nasally and are referred to as nasal chondromesenchymal hamartoma (NCMH). ${ }^{9}$ Infants and children are predominantly involved but it occurs in adults as well. It presents mainly with sleep-disordered breathing due to nasal obstruction, feeding difficulties in infants, recurrent sinusitis, serous otitis media, epistaxis and watery rhinorrhea, decreased sense of smell, or frontal headache with no nasal symptoms. Orbital involvement and intracranial expansion may result in additional severe symptoms. In 2015, a systematic review was identified among 48 cases in the literature in adults and children but only in the nasal or cranial region. All patients 
underwent operative resection of the NCMH. Although NCMH is a benign lesion, malignant transformation has been reported in the literature. ${ }^{4,9-15}$

The authors report the first case of chondromesenchymal hamartoma arising in ectopic thyroid tissue in neonate. Chondromesenchymal hamartomas usually arise from the nasal mucosa, a thyroid chondromesenchymal hamartoma is an exceedingly rare lesion, reported only in a few cases in the literature. ${ }^{16}$ No cases of ectopic thyroid tissue developing chondromesenchymal hamartoma have been reported, so far. Considering the wide extension of the hamartoma, MRI was performed for preoperative planning and complete surgical excision. The specimen revealed no evidence of malignant transformation.

Conflict of Interest

None.

\section{Acknowledgment}

The first author (A.SZ.) of the case report would like to thank Prof. Andrew B. Pinter for his support in writing and reviewing the article.

\section{References}

1 Guarisco JL. Congenital head and neck masses in infants and children. Part II. Ear Nose Throat J 1991;70(02):75-82

2 Azouz H, Salah H, Al-Ajlan S, Badran M. Treatment of cystic hygroma in a young infant through multidisciplinary approach involving sirolimus, sclerotherapy, and debulking surgery. JAAD Case Rep 2016;2(04):350-353

3 Lazar DA, Olutoye OO, Moise KJ Jr., et al. Ex-utero intrapartum treatment procedure for giant neck masses-fetal and maternal outcomes. J Pediatr Surg 2011;46(05):817-822

4 Rahbar R, Rodriguez-Galindo C, Meara JG, Smith ER, Perez-Atayde AR. Pediatric Head and Neck Tumors. A-Z Guide to Presentation and Multimodality Management. New York, NY: Springer-Verlag; 2014

5 Yap CY, Lin LH, Wang NK. An atypical presentation of Kawasaki disease: a 10-year-old boy with acute exudative tonsillitis and bilateral cervical lymphadenitis. Clinics (São Paulo) 2012;67(06): 689-692

6 Meier JD, Grimmer JF. Evaluation and management of neck masses in children. Am Fam Physician 2014;89(05):353-358

7 Helman LJ, Malkin D. Cancers of the childhood. In: DeVita VT Jr., Lawrence TS, Rosenberg SA, eds. DeVita, Hellman, and Rosenberg's Cancer: Principles and Practice of Oncology. 9th ed. Philadelphia, PA: Lippincott Williams \& Wilkins; 2011

8 Guerra G, Cinelli M, Mesolella M, et al. Morphological, diagnostic and surgical features of ectopic thyroid gland: a review of literature. Int J Surg 2014;12(Suppl 1):S3-S11

9 Mason KA, Navaratnam A, Theodorakopoulou E, Chokkalingam PG. Nasal Chondromesenchymal Hamartoma (NCMH): a systematic review of the literature with a new case report. J Otolaryngol Head Neck Surg 2015;44:28

10 Avcı H, Çomoğlu Ş, Öztürk E, Bilgiç B, Kıyak ÖE. Nasal chondromesenchymal hamartoma: a rare nasal benign tumor. Kulak Burun Bogaz Ihtis Derg 2016;26(05):300-303

11 Lee $\mathrm{CH}$, Park YH, Kim JY, Bae JH. Nasal chondromesenchymal hamartoma causing sleep-disordered breathing in an infant. Int J Clin Exp Pathol 2015;8(08):9643-9646

12 Uzomefuna V, Glynn F, Russell J, McDermott M. Nasal chondromesenchymal hamartoma with no nasal symptoms. BMJ Case Rep 2012;2012:bcr1120125148

13 Ünal A, Kum RO, Avc1 Y, Ünal DT. Nasal chondromesenchymal hamartoma, a rare pediatric tumor: Case report. Turk J Pediatr 2016;58(02):208-211

14 Li Y, Yang QX, Tian XT, Li B, Li Z. Malignant transformation of nasal chondromesenchymal hamartoma in adult: a case report and review of the literature. Histol Histopathol 2013;28(03):337-344

15 Chandra M, Sharma N, Venkatahalam VP. Nasal chondromesenchymal hamartoma: a case report and review of literature. JK Pract 2014;19:53-59

16 Chahal AS, Subramanyam CSV, Bhattacharjea AK. Chondromatous hamartoma of the thyroid gland: report of a case. Aust N Z J Surg 1975;45(01):30-31 